\title{
Effects of stiffness on the flow behavior of polymers
}

\author{
Arti Dua and Binny J. Cherayil \\ Department of Inorganic and Physical Chemistry, Indian Institute of Science, Bangalore-560012, India
}

\begin{abstract}
A recent model of the behavior of Gaussian chains in steady shear flow [J. Chem. Phys. 112, 8707 (2000)] is extended to include the effects of stiffness and finite extensibility. Calculations of the shear rate dependence of fractional elongation and of the time dependence of size fluctuations are found to be in good agreement with results from an experimental study of the behavior of single chains of DNA in steady shear flow. As in the earlier approach to the polymer-flow problem, we have ignored excluded volume and hydrodynamic interactions, but have instead added a bending energy contribution to the Hamiltonian of the chain, and have treated the usual connectivity term as a contribution to chain stretching that can be adjusted to ensure that the average size of the chain is fixed. The inclusion of stiffness and finite extensibility in the present treatment is found to produce significant improvements over the approach based purely on flexible chains.
\end{abstract}

\section{INTRODUCTION}

Flexible polymers can usually be modeled satisfactorily by random or self-avoiding walks, but stiff polymerspolyelectrolytes, for example - are not always so simply described. Flexible polymers themselves are not necessarily best represented as random walks if they are subject to forces that produce significant chain elongation. ${ }^{1}$ Increasingly, as interest in the control of polymer response to external perturbations grows alongside developments in the micromanipulation of single chains, ${ }^{2}$ it is often such forces that are deployed to monitor or modify chain conformational behavior. Several recent experimental and simulation papers have explored the nature of these conformational changes in some detail. ${ }^{3,4}$ The paper by Smith et al., ${ }^{3}$ for instance, is a study of the response of single molecules of very large DNA to steady shear flow. The DNA in these experiments are flexible to begin with but when subject to shear, they adopt quite extended conformations, although they never reach full extension under the conditions of the experiment. Chain extension under flow is not a new phenomenon, ${ }^{5}$ but advances in experimental methodology have brought greater refinement to its study. For instance, many of the distinct stages by which a compact configuration unfolds under shear can now be discerned in molecular detail. ${ }^{3,6}$ The regression of fluctuations around steady state configurations can also be measured ${ }^{3}$ it is found to occur with a characteristic rate that is related to the longest relaxation time of the chain.

If conventional descriptions of polymers based on flexible chain models are expected to fail when strong forces are present, understanding the results of the Smith et al. experiments, and others like them, is likely to require some understanding of the role of stiffness in chain dynamics. This is in fact borne out by recent work from our group that aimed at explaining the data from precisely these experiments using a Gaussian (i.e., a flexible) model of DNA conformations. ${ }^{7}$ The model's success was modest at best, but when it was modified to limit the maximum allowed extension (essen- tially by making the spring constant shear-dependent along the lines of FENE models ${ }^{8}$ ) the improvements were significant. However, the modifications were ad hoc, and stiffness was not treated at all. So although the model proved to be useful, and to have nice analytical properties, it lacked a convincing physical and mathematical foundation.

To do better, therefore, one needs to treat both stiffness and finite extensibility more rigorously. This is what we attempt to do in the present paper, which is a continuation of our efforts to provide a theoretical basis for understanding polymer-flow problems in general, and for rationalizing the results of the Smith et al. study in particular. The overall approach that we take here is much the same as before, in that the point of departure for describing chain dynamics in flow is the set of coupled stochastic equations for polymer and solvent that were introduced and developed by Oono, Freed, and their co-workers. ${ }^{9}$ The principal differences between the two studies lie in the nature of the chain Hamiltonian, which now explicitly includes terms associated with bending energies, and in the manner of accounting for finite extensibility, which now dispenses with the earlier FENE approximation. The present approach therefore treats the polymer as a continuous semiflexible chain.

The following section presents the equations that govern the dynamics of such semiflexible chains in shear flow, and provides their solution in terms of an expansion in a complete set of modes. These modes are generalizations of the Rouse modes that characterize the dynamics of flexible polymers, and contain a phenomenological parameter $z$ that is a measure of the rigidity of the chain. Large values of $z$ correspond to flexible polymers, while small values of $z$ correspond to stiff polymers. Section III describes the derivation of expressions for certain correlation functions that can be measured experimentally. Section IV compares the predicted values of these quantities with experiment, and discusses the implications of the results. A brief mathematical appendix furnishes details of intermediate steps in the derivation of certain results used in the text. 


\section{EVOLUTION OF CHAIN CONFORMATIONS UNDER FLOW}

At any instant of time $t$, and in units of the thermal energy $k_{B} T$, the Hamiltonian $H$ of a continuous semiflexible chain of contour length $N$ can be written as ${ }^{10-13}$

$$
H=\nu \int_{-N / 2}^{N / 2} d \tau\left|\frac{\partial \mathbf{r}(\tau, t)}{\partial \tau}\right|^{2}+\frac{\epsilon}{2} \int_{-N / 2}^{N / 2} d \tau\left|\frac{\partial^{2} \mathbf{r}(\tau, t)}{\partial \tau^{2}}\right|^{2},
$$

where $\mathbf{r}(\tau, t)$ is the spatial location of the monomer segment at a distance $\tau$ (as measured along the backbone) from the midpoint of the chain. This choice of origin for the points $\tau$ is a matter of convenience that simplifies some of the later algebra, but the more conventional choice places the origin at one end of the chain. The parameter $\epsilon$ is a phenomenological constant that is a measure of the bending energy of the chain, while $\nu$ is a parameter that can be interpreted as a measure of the stretching energy. In flexible chain models, $\epsilon$ is set to 0 , and $\nu$ is assigned the value $3 / 2 l$, where $l$ is the effective bond length. In semiflexible chain models, $\epsilon$ is nonzero, and can be written as $3 / 4 p$ (see Ref. 13, for example), where $p$, defined as $1 / l$, is half the inverse of the persistence length. When $\epsilon$ is nonzero, the vector $\partial \mathbf{r}(\tau, t) / \partial(\tau)$ must, strictly speaking, be regarded as a unit vector (as dictated by differential geometry), and the first term in $H$ should be absent, but it proves convenient to retain it, and to regard $\nu$ as a parameter that can be adjusted so that $|\dot{\mathbf{r}}(\tau)|$ is unity on average. In satisfying this constraint, the chain becomes essentially inextensible (i.e., its average contour length is $N$, but there can be fluctuations around this value).

If $H$ in Eq. (1) is regarded as the potential energy of the chain, its Lagrangian $\mathcal{L}$ is given by $\mathcal{L}$ $=(m / 2) \int_{-N / 2}^{N / 2} d \tau \dot{\mathbf{r}}(\tau, t)^{2}-H$, where $m$ is the monomer mass, and $\dot{\mathbf{r}}(\tau, t) \equiv \partial \mathbf{r}(\tau, t) / \partial t$ is the velocity of the segment at $\tau$. Then from the relation $(d / d t) \delta \mathcal{L} / \delta \mathbf{r}(\tau, t)=\delta \mathcal{L} / \delta \mathbf{r}(\tau, t)$, the equation of motion of the monomer becomes ${ }^{13}$

$$
\left(m \frac{\partial^{2}}{\partial t^{2}}+\epsilon \frac{\partial^{4}}{\partial \tau^{4}}-2 \nu \frac{\partial^{2}}{\partial \tau^{2}}\right) \mathbf{r}(\tau, t)=0,
$$

which must satisfy the following boundary conditions

$$
\begin{aligned}
& \left.\frac{\partial^{2} \mathbf{r}(\tau, t)}{\partial \tau^{2}}\right|_{\tau= \pm N / 2}=0 \\
& \epsilon \frac{\partial^{3} \mathbf{r}(\tau, t)}{\partial \tau^{3}}-\left.2 \nu \frac{\partial \mathbf{r}(\tau, t)}{\partial \tau}\right|_{\tau= \pm N / 2}=0 .
\end{aligned}
$$

In the overdamped limit of interest here, the inertial term in Eq. (2) may be neglected. Dissipation is then be accounted for by inclusion of a stochastic force that generates friction, while flow effects of the solvent are accounted for by inclusion of a systematic flow term and a fluctuating solvent velocity field. The final equation for monomer dynamics in flow thus becomes ${ }^{7,9}$

$$
\begin{aligned}
\left(\frac{\partial}{\partial t}\right. & \left.+\frac{\epsilon}{\zeta} \frac{\partial^{4}}{\partial \tau^{4}}-\frac{2 \nu}{\zeta} \frac{\partial^{2}}{\partial \tau^{2}}-\dot{\gamma} \mathbf{A} \cdot\right) \mathbf{r}(\tau, t) \\
& =\mathbf{v}(\mathbf{r}(\tau, t), t)+\theta(\tau, t),
\end{aligned}
$$

where $\zeta$ is the friction coefficient per unit length of the polymer, $\dot{\gamma}$ and $\mathbf{A}$ are, respectively, the strain rate and the velocity gradient tensor of the applied flow, $\mathbf{v}(\mathbf{r}, t)$ is the fluctuating solvent velocity field at the point $\mathbf{r}$ at time $t$, and $\boldsymbol{\theta}(\tau, t)$ is a random force with white noise statistics whose correlations satisfy

$$
\left\langle\boldsymbol{\theta}(\tau, t) \boldsymbol{\theta}\left(\tau^{\prime}, t^{\prime}\right)\right\rangle=\frac{2}{\zeta} \delta\left(\tau-\tau^{\prime}\right) \delta\left(t-t^{\prime}\right) \mathbf{I},
$$

I being the unit tensor. The term $\dot{\gamma} \mathbf{A}$-the systematic flow term-describes the velocity field of the solvent in the absence of the polymer, while the term $\mathbf{v}(\mathbf{r}(\tau, t), t)$ describes the fluctuations of the solvent velocity around the systematic flow in the presence of the polymer, and represents the coupling between the monomer at $\mathbf{r}(\tau, t)$ and the solvent at the same point. The field $\mathbf{v}(\mathbf{r}, t)$ itself is assumed to evolve according to the linearized Navier-Stokes equation, and leads to solvent-mediated hydrodynamic interactions (represented by the Oseen tensor) between different parts of the chain. ${ }^{9}$ For the present, as a simplifying approximation, these interactions will be neglected, so the term $\mathbf{v}(\mathbf{r}, t)$ in Eq. (5) may be dropped. The seriousness of this approximation is discussed in Sec. IV. [The complete set of polymer-solvent kinetic equations and their solution in terms of the Oseen tensor are provided in Ref. 7. Reference 14 shows how a combined multiple scattering renormalization group approach may used to treat hydrodynamic interactions perturbatively. These calculations are highly nontrivial, however, so their application to the flow behavior of semiflexible polymers will be deferred for future work.]

Equation (5), with $\mathbf{v}(\mathbf{r}, t)$ set to 0 , is seen to be solved by

$$
\begin{aligned}
r_{i}(\tau, t)= & \sum_{j} \int_{-N / 2}^{N / 2} d \tau_{1} G_{i j}\left(\tau, \tau_{1} \mid t\right) r_{j}\left(\tau_{1}\right) \\
& +\sum_{j} \int_{-N / 2}^{N / 2} d \tau_{1} \int_{0}^{t} d t_{1} G_{i j}\left(\tau, \tau_{1} \mid t-t_{1}\right) \theta_{j}\left(\tau_{1}, t_{1}\right),
\end{aligned}
$$

$i=x, y, z$,

where $\mathbf{r}(\tau)$ is the time $t=0$ position of the monomer at $\tau$, and $x, y, z$ are Cartesian components of the various vectorial quantities. The Green's function $G_{i j}$ is the solution to the following equation:

$$
\begin{aligned}
\sum_{j} & \left(\delta_{i j}\left[\frac{\partial}{\partial t}+\frac{\epsilon}{\zeta} \frac{\partial^{4}}{\partial \tau^{4}}-\frac{2 \nu}{\zeta} \frac{\partial^{2}}{\partial \tau^{2}}\right]-\dot{\gamma} A_{i j}\right) G_{j k}\left(\tau, \tau_{1} \mid t\right) \\
& =\delta_{i k} \delta\left(\tau-\tau_{1}\right) \delta(t),
\end{aligned}
$$

and is given by

$$
G_{j k}\left(\tau, \tau_{1} \mid t\right)=\left[e^{\dot{\gamma} \mathbf{A} t}\right]_{j k} G_{0}\left(\tau, \tau_{1} \mid t\right),
$$

where $G_{0}$-the unperturbed Green's function-is in turn the solution to

$$
\left(\frac{\partial}{\partial t}+\frac{\epsilon}{\zeta} \frac{\partial^{4}}{\partial \tau^{4}}-\frac{2 \nu}{\zeta} \frac{\partial^{2}}{\partial \tau^{2}}\right) G_{0}\left(\tau, \tau_{1} \mid t\right)=\delta\left(\tau-\tau_{1}\right) \delta(t) .
$$

By direct substitution, Eq. (10) is seen to be solved by 


$$
G_{0}\left(\tau, \tau_{1} \mid t\right)=\theta(t) \sum_{n=0}^{\infty} Q_{n}(\tau) Q_{n}\left(\tau_{1}\right) e^{-\lambda_{n} t / \zeta},
$$

where $\theta(t)$ is the step function. $Q_{n}(\tau), n=0,1,2, \ldots$ form a complete orthonormal set of eigenfunctions that are the solutions to the following eigenvalue equation:

$$
L Q_{n}(\tau)=\lambda_{n} Q_{n}(\tau), \quad n=0,1,2, \ldots,
$$

where $\lambda_{n}$ are the eigenvalues, and $L$ is the Hermitian operator,

$$
L=\epsilon \frac{\partial^{4}}{\partial \tau^{4}}-2 \nu \frac{\partial^{2}}{\partial \tau^{2}} .
$$

The eigenfunctions $Q_{n}(\tau)$ satisfy the same boundary conditions as defined in Eqs. (3) and (4). In terms of the eigenvalues $\lambda_{n}$, a set of characteristic relaxation times $\tau_{n}$ for the various modes in Eq. (11) may be defined as $\tau_{n}=\zeta / \lambda_{n}$.

Equation (12) is a simple fourth order differential equation that may be solved by rewriting it as a system of four first order differential equations in a new set of dependent variables. ${ }^{15}$ In matrix notation, this system is of the form $\partial \mathbf{X}(\tau) / \partial \tau=\mathbf{M} \cdot \mathbf{X}(\tau)$. From the solution to this equation, one can easily show that

$$
\begin{aligned}
Q_{n}(\tau)= & A \cos \left(\beta_{n} \tau\right)+B \cosh \left(\alpha_{n} \tau\right)+C \sin \left(\beta_{n} \tau\right) \\
& +D \sinh \left(\alpha_{n} \tau\right),
\end{aligned}
$$

where $A, B, C$, and $D$ are constants to be determined from the boundary conditions, and $\alpha_{n}$ and $\beta_{n}$ are parameters that are obtained from the characteristic equation of the matrix $\mathbf{M}$. They are defined as

$$
\begin{aligned}
& \alpha_{n}=\left[\frac{\nu}{\epsilon} \pm\left[\left(\frac{\nu}{\epsilon}\right)^{2}+\frac{\lambda_{n}}{\epsilon}\right]^{1 / 2}\right]^{1 / 2}, \\
& i \beta_{n}=-\left[\frac{\nu}{\epsilon} \mp\left[\left(\frac{\nu}{\epsilon}\right)^{2}+\frac{\lambda_{n}}{\epsilon}\right]^{1 / 2}\right]^{1 / 2} .
\end{aligned}
$$

$\alpha_{n}$ and $\beta_{n}$ are not independent, but are related to each other and to the eigenvalues $\lambda_{n}$ by the equations

$$
\alpha_{n}^{2}-\beta_{n}^{2}=\frac{2 \nu}{\epsilon}
$$

and

$$
\lambda_{n}=\alpha_{n}^{2} \beta_{n}^{2} \epsilon .
$$

The fact that $Q_{n}(-\tau)$ also satisfies Eq. (12) means that the linear combinations $Q_{n}(\tau)+Q_{n}(-\tau)$ and $Q_{n}(\tau)-Q_{n}(-\tau)$ are also solutions, so the eigenfunctions may be chosen with definite parity. In general, then, they are given by $Q_{n}(\tau)$ $=A \cos \left(\beta_{n} \tau\right)+B \cosh \left(\alpha_{n} \tau\right)$ (even parity solutions), and by $Q_{n}(\tau)=C \sin \left(\beta_{n} \tau\right)+D \sinh \left(\alpha_{n} \tau\right)$ (odd parity solutions). For $n \geqslant 1$, the application of the boundary conditions in Eq. (3) readily determines these solutions as

$$
\begin{aligned}
Q_{n}(\tau)= & \left(\frac{C_{n}}{N}\right)^{1 / 2}\left[\frac{\cos \left(\beta_{n} \tau\right)}{\cos \left(\beta_{n} N / 2\right)}\right. \\
& \left.+\left(\frac{\beta_{n}}{\alpha_{n}}\right)^{2} \frac{\cosh \left(\alpha_{n} \tau\right)}{\cosh \left(\alpha_{n} N / 2\right)}\right], \quad \text { (even parity), }
\end{aligned}
$$

$$
\begin{aligned}
Q_{n}(\tau)= & \left(\frac{D_{n}}{N}\right)^{1 / 2}\left[\frac{\sin \left(\beta_{n} \tau\right)}{\sin \left(\beta_{n} N / 2\right)}\right. \\
& \left.+\left(\frac{\beta_{n}}{\alpha_{n}}\right)^{2} \frac{\sinh \left(\alpha_{n} \tau\right)}{\sinh \left(\alpha_{n} N / 2\right)}\right], \quad \text { (odd parity), }
\end{aligned}
$$

where $C_{n}$ and $D_{n}$ are unknown but can be chosen to ensure normalization of the eigenfunctions. The further application of the boundary conditions in Eq. (4) leads to a pair of eigenvalue equations that correspond to the even and odd parity solutions; these equations are

$$
\begin{aligned}
& \alpha_{n}^{3} \sinh \left(\alpha_{n} N / 2\right) \cos \left(\beta_{n} N / 2\right) \\
& \quad-\beta_{n}^{3} \cosh \left(\alpha_{n} N / 2\right) \sin \left(\beta_{n} N / 2\right)=0, \\
& \alpha_{n}^{3} \cosh \left(\alpha_{n} N / 2\right) \sin \left(\beta_{n} N / 2\right) \\
& \quad+\beta_{n}^{3} \sinh \left(\alpha_{n} N / 2\right) \cos \left(\beta_{n} N / 2\right)=0,
\end{aligned}
$$

and they may be combined into the single compact form

$$
\begin{gathered}
1-\cos \left(\beta_{n} N\right) \cosh \left(\alpha_{n} N\right)+\frac{1}{2}\left(\frac{\beta_{n}^{3}}{\alpha_{n}^{3}}-\frac{\alpha_{n}^{3}}{\beta_{n}^{3}}\right) \sin \left(\beta_{n} N\right) \\
\times \sinh \left(\alpha_{n} N\right)=0, \quad n=0,1,2, \ldots
\end{gathered}
$$

For $n=0$, corresponding to an eigenvalue of 0 , the eigenfunction $Q_{0}(\tau)$ is just a constant, so from the normalization condition, we have

$$
Q_{0}(\tau)=\frac{1}{\sqrt{N}} .
$$

\section{CALCULATION OF CORRELATION FUNCTIONS}

To make contact with experiment, we shall, as before, calculate two of the quantities measured in Ref. 3; the normalized time correlation function of the chain extension $C(s)$ as a function of a dimensionless time, and the mean fractional extension $\mu$ as a function of a dimensionless shear rate. Both quantities can be calculated from the following function, denoted $B(s)$ and defined as

$$
B(s) \equiv\langle\mathbf{R}(t) \cdot \mathbf{R}(t+s)\rangle .
$$

Here $s$ is some arbitrary interval of time, and $\mathbf{R}(t)$ is the end-to-end distance of the chain at time $t$, i.e.,

$$
\mathbf{R}(t) \equiv \mathbf{r}(N / 2, t)-\mathbf{r}(-N / 2, t) .
$$

The angular brackets denote an average with respect to the initial distribution of chain conformations. In the limit of long times, $t \rightarrow \infty$, this average is equivalent (as shown earlier ${ }^{7}$ ) to the steady state average that determines the measured values of experimental observables.

In terms of $B(s)$, the two quantities of interest, $C(s)$ and $\mu$, are given by

$$
\begin{aligned}
& C(s)=B(s) / B(0), \\
& \mu=\frac{1}{N} \sqrt{B(0)} .
\end{aligned}
$$

From Eqs. (25) and (26), one sees that $B(s)$ can be expressed as 


$$
\begin{aligned}
B(s)= & X(N / 2, N / 2 \mid t, t+s)-X(N / 2,-N / 2 \mid t, t+s) \\
& -X(-N / 2, N / 2 \mid t, t+s)+X(-N / 2,-N / 2 \mid t, t+s),
\end{aligned}
$$

where, in general,

$$
X\left(\tau, \tau^{\prime} \mid t, t+s\right)=\left\langle\mathbf{r}(\tau, t) \cdot \mathbf{r}\left(\tau^{\prime}, t+s\right)\right\rangle .
$$

$X\left(\tau, \tau^{\prime} \mid t, t+s\right)$ in turn, from Eq. (7), can be written as

$$
X\left(\tau, \tau^{\prime} \mid t, t+s\right)=X_{1}\left(\tau, \tau^{\prime} \mid t, t+s\right)+X_{2}\left(\tau, \tau^{\prime} \mid t, t+s\right),
$$

where

$$
\begin{aligned}
X_{1}\left(\tau, \tau^{\prime} \mid t, t+s\right)= & \sum_{i j k} \int_{-N / 2}^{N / 2} d \tau_{1} \int_{-N / 2}^{N / 2} d \tau_{2} G_{i j}\left(\tau, \tau_{1} \mid t\right) \\
& \times\left\langle r_{j}\left(\tau_{1}\right) r_{k}\left(\tau_{2}\right)\right\rangle G_{k i}\left(\tau^{\prime}, \tau_{2} \mid t+s\right)
\end{aligned}
$$

and

$$
\begin{aligned}
X_{2}\left(\tau, \tau^{\prime} \mid t, t+s\right)= & \sum_{i j k} \int_{-N / 2}^{N / 2} d \tau_{1} \int_{-N / 2}^{N / 2} d \tau_{2} \int_{0}^{t} d t_{1} \int_{0}^{t+s} d t_{2} \\
& \times G_{i j}\left(\tau, \tau_{1} \mid t-t_{1}\right)\left\langle\theta_{j}\left(\tau_{1}, t_{1}\right) \theta_{k}\left(\tau_{2}, t_{2}\right)\right\rangle \\
& \times G_{k i}\left(\tau^{\prime}, \tau_{2} \mid t+s-t_{2}\right) .
\end{aligned}
$$

From Eq. (29), $B(s)$ is now seen to reduce to a sum of terms involving $\quad X_{1} \quad\left[\right.$ such as, $X_{1}(N / 2, N / 2 \mid t, t+s), X_{1}(N / 2$, $-N / 2 \mid t, t+s)$, etc. $]$ and similar terms involving $X_{2}$. If these separate contributions are denoted $B_{1}(s)$ and $B_{2}(s)$, respectively, one can show for the case of simple shear flow, using Eqs. (9) and (11) and the results

$$
\mathbf{A}=\left(\begin{array}{lll}
0 & 1 & 0 \\
0 & 0 & 0 \\
0 & 0 & 0
\end{array}\right)
$$

and

$$
\operatorname{Tr}\left[\left(e^{\dot{\gamma} \mathbf{A} t_{1}}\right)\left(e^{\dot{\gamma} \mathbf{A} t_{2}}\right)^{T}\right]=3+\dot{\gamma}^{2} t_{1} t_{2},
$$

that

$$
\begin{aligned}
B_{1}(s)= & \left(3+\dot{\gamma}^{2} t(t+s)\right) \sum_{n=0}^{\infty}\left(Q_{n}(N / 2)\right. \\
& \left.-Q_{n}(-N / 2)\right)^{2} \exp \left(-\lambda_{n}(t+2 s) / \zeta\right) \\
& \times \int_{-N / 2}^{N / 2} d \tau_{1} \int_{-N / 2}^{N / 2} d \tau_{2} \alpha\left(\tau_{1}, \tau_{2}\right) Q_{n}\left(\tau_{1}\right) Q_{n}\left(\tau_{2}\right),
\end{aligned}
$$

where the function $\alpha\left(\tau_{1}, \tau_{2}\right)$ is defined by the relation $\left\langle r_{j}\left(\tau_{1}\right) r_{k}\left(\tau_{2}\right)\right\rangle=\delta_{j k} \alpha\left(\tau_{1}, \tau_{2}\right)$. The precise form of $\alpha\left(\tau_{1}, \tau_{2}\right)$ is readily determined, but is not needed, as one can show that in the long time limit of interest, viz., $t \rightarrow \infty, B_{1}(s)$ vanishes. This leaves $B_{2}(s)$ as the sole contribution to $B(s) . B_{2}(s)$, from Eqs. (33), (9), and (11), is given by

$$
\begin{aligned}
B_{2}(s)= & \frac{2}{\zeta} \sum_{n=0}^{\infty}\left(Q_{n}(N / 2)-Q_{n}(-N / 2)\right)^{2} e^{-\lambda_{n} s / \zeta} \int_{0}^{t} d t_{1} \\
& \times\left[3+\dot{\gamma}^{2}\left(t-t_{1}\right)\left(t+s-t_{1}\right)\right] e^{-2 \lambda_{n}\left(t-t_{1}\right) / \zeta} .
\end{aligned}
$$

The integral over $t_{1}$ in this expression is easily carried out. The result, when $t$ is set to infinity, leads to

$$
\begin{aligned}
C(s)=\frac{B_{2}(s)}{B_{2}(0)}= & \frac{1}{B_{2}(0)} \sum_{n=1}^{\infty}\left[\frac{3}{\lambda_{n}}+\frac{\dot{\gamma}^{2}}{2}\left(\frac{s \zeta}{\lambda_{n}^{2}}+\frac{\zeta^{2}}{\lambda_{n}^{3}}\right)\right] \\
& \times e^{-\lambda_{n} s / \zeta}\left(Q_{n}(N / 2)-Q_{n}(-N / 2)\right)^{2} .
\end{aligned}
$$

As a further simplifying approximation, we shall neglect all but the lowest eigenmode in this equation (contributions from the higher modes can be shown to be small), and rewrite the resulting expression in terms of the dimensionless parameters that are used in the data of Smith et al. ${ }^{3}$ These parameters are the reduced time $s^{*} \equiv \dot{\gamma} s$, and the Weissenberg number $\mathrm{Wi}$, defined as the product of the shear rate $\dot{\gamma}$ and the longest relaxation time $\tau$ of the DNA molecule. $\tau$ is obtained from measurements of the unperturbed chain (which is largely flexible under the conditions of the experiment), so it is reasonable to assume that $\tau$ may be identified with the Rouse relaxation time. In units where $k_{B} T=1$, this means

$$
\tau=\frac{\zeta N^{2} l}{3 \pi^{2}} \equiv \frac{\zeta N^{2}}{3 \pi^{2} p} .
$$

As stated earlier, $l$ is the bond length, (recall that $\zeta$ is the friction coefficient per unit length of the chain), and $p$ is $1 / l$. In our earlier treatment of chain dynamics in shear flow, Ref. 7 , we used units where $l / d, d$ being the dimension of space, was set to unity; $\tau$ in that reference was therefore defined as $\zeta N^{2} / \pi^{2}$. A dimensionless measure of persistence $z \equiv p N$, having the property that the limit $z \gg 1$ describes flexible chains and the limit $z \ll 1$ describes stiff chains, is now introduced into Eq. (38), along with the definitions $s^{*}=\dot{\gamma} s$ and $\mathrm{Wi}=\dot{\gamma} \tau$, to rewrite it in the dimensionless form

$$
C(s)=\left[1+\frac{\pi^{2} z s^{*} \mathrm{Wi} / 2 \lambda_{1} N^{3}}{\left(1+3 \pi^{4} z^{2} \mathrm{Wi}^{2} / 2 \lambda_{1}^{2} N^{6}\right)}\right] \exp \left(-\frac{\lambda_{1} s^{*} N^{3}}{3 \pi^{2} z \mathrm{Wi}}\right) .
$$

From Eq. (28), the mean fractional extension can likewise be expressed in dimensionless form,

$$
\mu=\left[\frac{3}{\lambda_{1} N^{2}}+\frac{9 \pi^{4} z^{2} \mathrm{Wi}^{2}}{2 \lambda_{1}^{3} N^{8}}\right]^{1 / 2}\left(Q_{1}(N / 2)-Q_{1}(-N / 2)\right) .
$$

While Eqs. (40) and (41) provide the desired expressions for the calculation of the two quantities of interest, they can be evaluated only after the eigenvalues and eigenfunctions defined by Eq. (12) are themselves calculated. To calculate these latter quantities, one needs to solve Eqs. (17), (18), and (20). However, these equations have to be supplemented by the equations that enforce inextensibility according to the prescription outlined briefly in Sec. II, and ignored up to now, so the eventual evaluation of $\lambda_{n}$ and $Q_{n}$ will involve solving a set of simultaneous transcendental equations, which can only be done numerically.

As stated earlier, the tangent vector $\partial \mathbf{r}(\tau) / \partial \tau \equiv \mathbf{u}(\tau)$ must be a unit vector for all $\tau$ according to the requirements of differential geometry. Enforcing this constraint in the present calculations is difficult, however, so we shall merely require that $\left\langle\mathbf{u}(\tau)^{2}\right\rangle=1$, where the brackets refer to an aver- 
age over all allowed chain conformations. This alternative constraint (introduced by Harris and Hearst ${ }^{12}$ ) is equivalent to fixing the average length of the chain at $N$, and it produces the correct scaling behavior of the end-to-end vector $\mathbf{R}$ in the coil and rod limits in the absence of shear. We shall make some further remarks about the Harris-Hearst procedure in Sec. IV.

An expression for the tangent vector $\mathbf{u}(\tau, t)$ can be obtained from Eq. (7) by differentiation with respect to $\tau$, in this way we find

$$
\begin{aligned}
u_{i}(\tau, t)= & \sum_{j} \int_{-N / 2}^{N / 2} d \tau_{1} \frac{\partial}{\partial \tau} G_{i j}\left(\tau, \tau_{1} \mid t\right) r_{j}\left(\tau_{1}\right) \\
& +\sum_{j} \int_{-N / 2}^{N / 2} d \tau_{1} \int_{0}^{t} d t_{1} \frac{\partial}{\partial \tau} G_{i j}\left(\tau, \tau_{1} \mid t, t_{1}\right) \theta_{j}\left(\tau_{1}, t_{1}\right) .
\end{aligned}
$$

By following the same sequence of steps as led to the expression for $X\left(\tau, \tau^{\prime} \mid t\right)$ [Eq. (30)], one can show that

$$
\left\langle\mathbf{u}(\tau, t)^{2}\right\rangle=\left\langle\mathbf{u}(\tau, t)^{2}\right\rangle_{0}+\left\langle\mathbf{u}(\tau, t)^{2}\right\rangle_{s},
$$

where $\left\langle\mathbf{u}(\tau, t)^{2}\right\rangle_{0}$ is the mean square tangent vector in the absence of shear, and $\left\langle\mathbf{u}(\tau, t)^{2}\right\rangle_{s}$ is the correction due to shear. $\left\langle\mathbf{u}(\tau, t)^{2}\right\rangle_{0}$ can be determined exactly using the Hamiltonian of Eq. (1) (the derivation is sketched in the Appendix), and it is given by

$$
\begin{aligned}
\left\langle\mathbf{u}(\tau, t)^{2}\right\rangle_{0} & \\
= & \left(\frac{9}{2 \nu \epsilon}\right)^{1 / 2} \\
& \times \frac{\cosh \left((2 \nu / \epsilon)^{1 / 2}(N / 2+\tau)\right) \cosh \left((2 \nu / \epsilon)^{1 / 2}(N / 2-\tau)\right)}{\sinh \left((2 \nu / \epsilon)^{1 / 2} N\right)} .
\end{aligned}
$$

The average $\left\langle\mathbf{u}(\tau, t)^{2}\right\rangle_{s}$ is given by

$$
\left\langle\mathbf{u}(\tau, t)^{2}\right\rangle_{s}=\sum_{n=1}^{\infty} \frac{9 \pi^{4} z^{2} \mathrm{Wi}^{2}}{2 \lambda_{n}^{3} N^{6}}\left|\frac{\partial Q_{n}(\tau)}{\partial \tau}\right|^{2} .
$$

After integrating Eqs. (44) and (45) over $\tau$ and setting the result to 1 , the following constraint equation is produced:

$$
\begin{aligned}
1= & \frac{3}{4 \nu N}\left[1+\left(\frac{2 \nu}{\epsilon}\right)^{1 / 2} N \operatorname{coth}\left(\left(\frac{2 \nu}{\epsilon}\right)^{1 / 2} N\right)\right] \\
& +\sum_{n=1}^{\infty} \frac{9 \pi^{4} z^{2} \mathrm{Wi}^{2} I_{n}}{2\left(\epsilon \beta_{n}^{4}+2 \nu \beta_{n}^{2}\right)^{3} N^{7}},
\end{aligned}
$$

where

$$
I_{n}=\int_{-N / 2}^{N / 2} d \tau\left|\frac{\partial Q_{n}(\tau)}{\partial \tau}\right|^{2}
$$

If the sum in Eq. (46) is restricted to the lowest mode, the constraint equation simplifies to

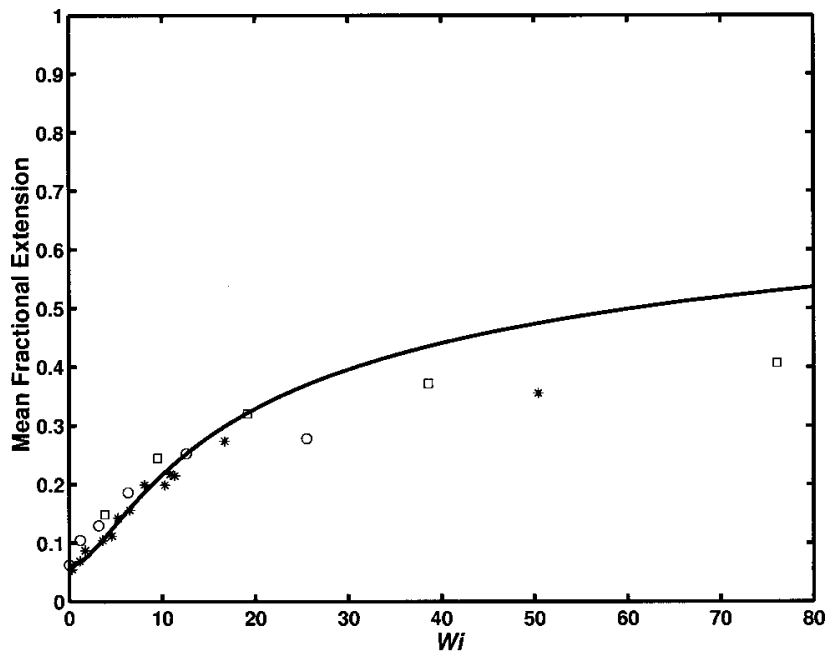

FIG. 1. Mean fractional extension versus Weissenberg number Wi. Open symbols are the data of Ref. 3 on the $\lambda$-phage DNA at two different viscosities: $60 \mathrm{cP}$ (open circles) and $220 \mathrm{cP}$ (open squares). Asterisks are data from the simulations of Ref. 16 on a 20 -unit bead-spring model. The full line is the theoretical curve calculated from Eq. (41) using a stiffness parameter $z$ of 220 .

$$
\begin{gathered}
\frac{3}{4 \nu N}\left[1+\left(\frac{2 \nu}{\epsilon}\right)^{1 / 2} N \operatorname{coth}\left(\left(\frac{2 \nu}{\epsilon}\right)^{1 / 2} N\right)\right] \\
+\frac{9 \pi^{4} z^{2} \mathrm{Wi}^{2} I_{1}}{2\left(\epsilon \beta_{1}^{4}+2 \nu \beta_{1}^{2}\right)^{3} N^{7}}-1=0 .
\end{gathered}
$$

As mentioned earlier, $\nu$ is regarded as an adjustable parameter, so Eqs. (48) and (23) [along with Eq. (17)] are now solved simultaneously for the dimensionless variables $\beta_{1} N$ and $\nu N$ at selected values of the Weissenberg number $\mathrm{Wi}$ and of the stiffness parameter $z$ (which, it may be recalled, also enters into the parameter $\epsilon$ through the dimensionless combination $\epsilon / N=3 / 4 z$ ), and the results substituted into Eqs. (40) and (41) to calculate $C(s)$ and $\mu$. [The validity of the lowest mode approximation can be tested by truncating the series expansion for $\left\langle\mathbf{u}(\tau, t)^{2}\right\rangle_{s}$ at successively higher values of $n$, and solving for the increased number of unknown parameters by the method above. When the series is truncated at $n=2$, for instance, one has to solve for $\beta_{1}, \beta_{2}$, and $\nu$. For this case, we can show explicitly that the contribution to the sum from the $n=2$ mode is between two to three orders of magnitude smaller than the contribution from $n=1$. Contributions from the higher modes are expected to be smaller still. We believe, therefore, that lowest mode approximation is a good one.]

\section{RESULTS AND DISCUSSION}

\section{A. Mean fractional extension}

Both experiment ${ }^{3}$ and simulation ${ }^{16}$ have determined the extent of chain elongation as a function of the strength of the applied shear. The experimental measurements of Ref. 3 (reproduced in Fig. 1) show that as the shear rate increases, the chain quickly extends, but then assumes a nearly constant value of roughly half the maximum possible extension beyond a Weissenberg number of about 20. The measurements are not carried out beyond a Weissenberg number of 80 , so it 
is not clear whether this putative asymptotic behavior of the fractional extension persists, or whether full extension is at some point achieved. The simulation data of Ref. 16 are in very good agreement with these trends, at least up to a Weissenberg number of 80 , but there are clear indications from the data beyond $\mathrm{Wi}=80$ (which are not shown in Fig. 1) that chain extension continues thereafter, although slowly enough that full extension would seem to be a remote possibility for flows that remain smooth. It should be noted that the lengths of the chains in the two sets of data are quite different; the simulations are carried out on a bead-spring model of 20 beads, while the experiments are carried out on DNA of about 50000 base pairs.

Our theoretical estimates of the mean fractional extension $\mu$ are obtained from Eq. (41), which for a given value of Wi depends on three additional parameters: the dimensional inverse Kuhn length $z$, the eigenvalue $\lambda_{1}$, and the eigenfunction $Q_{1} . z$ is a measure of the persistence length of the chain, and is in general not known a priori, though it can be determined from experiment or simulation. Smith et al. ${ }^{3}$ estimate the number of persistence lengths in their shear-free sample of DNA to be 440, so $z$ may be assigned this value in the present calculations. However, there is a factor 2 difference between the definitions of the persistence lengths in the two studies, and the value of $z$ that actually needs to be used in our calculations is 220. Fixing $z$ at this value (thus assigning a definite value to $\epsilon / N$, which is equal to $3 / 4 z$ ), we next solve-numerically - the simultaneous Eqs. (17), (23), and (48) for the dimensionless variables $\beta_{1} N$ and $\nu N$ for different chosen values of Wi. The results are then used in Eqs. (18) and (20) to determine $\lambda_{1}$ and $Q_{1}$ at the chosen values of Wi. These results in turn are finally used in Eq. (41) to obtain $\mu$ as a function of Wi at the given initial value of $z$. The calculated variation of $\mu$ with Wi is shown as the full curve in Fig. 1, and it is seen to agree qualitatively with the results of experiment and simulation. The agreement is especially good at the lower end of the Wi scale, and the slow approach to a possibly asymptotic limit at very high shear rates is reproduced. The predicted value of this limit is somewhat higher than the observed value, which may or may not represent a point of discrepancy, given the difficulty in making accurate measurements of chain size at high shear rates.

\section{B. Decay of size fluctuations}

Reference 3 also provides data on the steady state decay of fluctuations in the chain extension for different fixed values of Wi. Three such sets of data points (normalized to unity at the initial time) are shown in Fig. 2. The fluctuations are seen to regress very quickly at small $\mathrm{Wi}$, when the chain is expected to be still flexible, and much more slowly at large $\mathrm{Wi}$, when the chain is more stiff. The corresponding theoretical decay curves $C(s)$ are calculated from Eq. (40), after solving Eqs. (17), (23), and (48) for $\beta_{1} N$ and $\nu N$ at chosen values of $z$ and $\mathrm{Wi}$, along much the same lines as the calculation of $\mu$. In that calculation, however, $z$ is the stiffness parameter of the chain before it is subjected to flow, and can be assigned the value determined by measurements on the unperturbed chain. The experimental measurements of $C(s)$, on the other hand, are reported for fixed nonzero values of

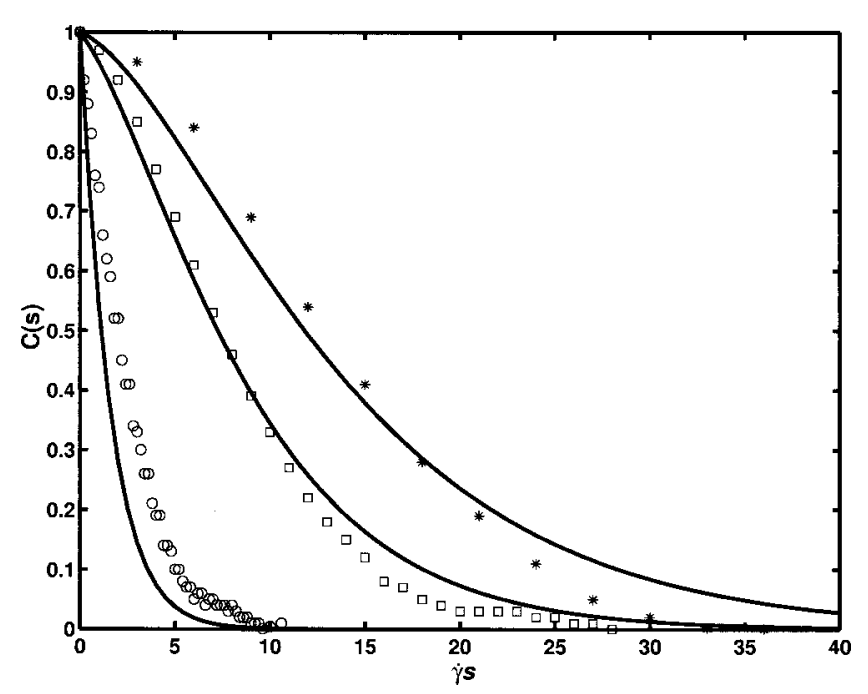

FIG. 2. Normalized time correlation function of the chain extension vs the dimensionless time. Data points are from the measurements of Ref. 3 on $\lambda$-phage DNA for three different fixed values of Wi at constant viscosity. Open circles correspond to $\mathrm{Wi}=1.3$, open squares to $\mathrm{Wi}=6.3$, and asterisks to $\mathrm{Wi}=19$. The full lines are the theoretical decay curves calculated from Eq. (40) for different fixed values of $z$. The $z$ values are 110, 30.7, and 10.5 corresponding to $\mathrm{Wi}=1.3,6.3$, and 19 , respectively.

Wi (specifically, for $\mathrm{Wi}=1.3,6.3$, and 19), and at these shear rates, $z$ can be expected to differ from the value of 220 it assumes under conditions of no shear. No values of $z$ for these Wi are available from experiment, nor can they be determined, in general, from the present formalism. We have therefore estimated them as follows: from the experimental variation of $\mu$ with Wi (Fig. 1), we obtain the values of the fractional extensions that correspond to the given values of Wi $(1.3,6.3$, and 19). Our estimates of the fractional extensions for these values of $\mathrm{Wi}$ are $0.095,0.18$, and 0.30 , respectively. We then use the well-known Kratky-Porod result for the end-to-end distance of a semiflexible polymer in the form

$$
\mu_{\mathrm{KP}} \equiv \frac{\left\langle R^{2}\right\rangle^{1 / 2}}{N}=\frac{1}{\sqrt{z}}\left[1-\frac{1}{2 z}\left(1-e^{-2 z}\right)\right]^{1 / 2}
$$

to determine, from the graph of $\mu_{\mathrm{KP}}$ vs $z$ the values of $z$ that correspond to the fractional extensions that have just been determined $(0.095,0.18$, and 0.30$)$. We estimate these values of $z$ to be $110,30.7$, and 10.5 , respectively (confirming, as anticipated, that the chain is becoming progressively less flexible, although it is still far from being completely rigid). We now use these values of $z$, along with the corresponding Wi (1.3, 6.3, and 19, respectively) to solve Eqs. (23) and (48) [using Eq. (17)], and thence to solve Eq. (40) for $C(s)$ as a function of $\dot{\gamma} s . C(s)$ calculated in this way is shown as the solid curves in Fig. 2.

The results are in very satisfactory agreement with the experimental data for $\mathrm{Wi}=6.3$ and 19 , and suggest that at the present level of approximation (neglect of hydrodynamic and excluded volume interactions), the effects of stiffness and finite extensibility on the behavior of polymers in flow are significant. At $\mathrm{Wi}=1.3$, however, there are more pronounced deviations between the experimental and theoretical curves. This would seem to suggest that the neglect of hydrodynamic 
and excluded volume interactions in the calculations carried out at this flow rate is less benign, but the reasons for the deviations may be more complex, as hydrodynamic interactions (if not necessarily excluded volume interactions) are potentially important even at higher flow rates. A calculation by $\mathrm{Zimm}^{17}$ of the mean extension of a wormlike chain under flow in the presence of hydrodynamic interactions compares well with experimental data on the stretching of tethered DNA in extensional flow, even at the highest flow rates, where the chains are stretched up to $80 \%$ of full extension. However, it is by no means clear that the success of the model can be ascribed to the inclusion of hydrodynamic interactions alone, as the model also includes limits on the maximum possible extension under the action of a force through a six-parameter rational approximation formula that can be seen as the analog of the constraint equation used in our calculations. There is no obvious way to distinguish the effects of finite extensibility from the effects of hydrodynamic interactions.

A very recent calculation by Wang and Chatterjee ${ }^{18}$ of extension vs flow for the system polystyrene in dioctyl phthalate generalizes the FENE approach developed in our earlier model ${ }^{7}$ to include hydrodynamic interactions within the preaveraged Zimm approximation. In the absence of the finite extensibility constraint, both the Rouse and Zimm models are found to overestimate the extent of stretching, but the agreement between theory and experiment is significantly improved, especially after the incorporation of hydrodynamic interactions, once this constraint is accounted for. As argued earlier, ${ }^{7}$ however, the FENE approach, despite its simplicity, represents an uncontrolled approximation of uncertain validity that in general is not easy to correct systematically. The approximation can be avoided by treating hydrodynamic interactions along the lines of Harnau et al.'s treatment of the dynamic structure factor of semiflexible chains. ${ }^{19}$ But preaveraging is still necessary, and this is yet another uncontrolled approximation that at least for certain dynamical properties can introduce errors of as much as $10 \%-15 \% .{ }^{20}$ On the other hand, the approximation appears to provide a perfectly consistent description of experiments by Quake et al. ${ }^{21}$ on the dynamics of partially extended single molecules of DNA undergoing Brownian motion. While these results are suggestive, they do not provide a direct test of the utility of the approximation under conditions of flow. The preaveraging approximation need not be invoked in the approach that we have developed here, but the subsequent calculations then become quite involved (though still analytically tractable, for the most part).

There is one other respect in which the present formalism could be seen as having limitations, and that is its use of the Harris-Hearst model ${ }^{12}$ to enforce constancy of the contour length. The model does not correspond to a homogeneously stiff chain, for instance, and it fails adequately to describe chain dynamics in the rigid rod limit. ${ }^{22}$ The lack of homogeneity in the model was corrected by Lagowski et al. ${ }^{23}$ by the inclusion of terms in the Hamiltonian that suppressed unphysically large fluctuations of the monomers at the ends of the chain. Their results were rediscovered by Harnau et al. ${ }^{13}$ using a much less transparent maximum en- tropy formalism. While these modifications do correct for shortcomings in the physics of the Harris-Hearst model of the unperturbed chain, they fail to ensure finite extensibility when static forces are present, unless the parameters that account for chain end effects are freely adjusted. But then the model is little different from the Harris-Hearst model itself, and becomes much less easy to apply. It therefore proves convenient to use the Harris-Hearst approach from the outset. Moreover, in the context of the present calculations, which do not consider the limit of completely rigid chains, the approach is unlikely to introduce any serious errors.

\section{ACKNOWLEDGMENTS}

We are grateful to Hazen Babcock, Stanford University, for helpful discussions on the behavior of polymers in flow. We would also like to thank Avik Chatterjee, SUNY, Syracuse, for a preprint of his work on shear flow.

\section{APPENDIX: CALCULATION OF THE MEAN SQUARE TANGENT VECTOR}

From the definition

$$
\begin{aligned}
K\left(\mathbf{u}_{f}, \mathbf{u}_{i} ; N / 2,-N / 2\right)= & \int_{\mathbf{u}(-N / 2)=\mathbf{u}_{i}}^{\mathbf{u}(N / 2)=\mathbf{u}_{f}} D[\mathbf{u}(\tau)] \\
& \times \exp [-S[\mathbf{u}]],
\end{aligned}
$$

where

$$
S[\mathbf{u}]=\nu \int_{-N / 2}^{N / 2} d \tau|\mathbf{u}(\tau)|^{2}+\frac{\epsilon}{2} \int_{-N / 2}^{N / 2} d \tau\left|\frac{\partial \mathbf{u}(\tau)}{\partial \tau}\right|^{2},
$$

the average $\left\langle\mathbf{u}^{2}(\tau)\right\rangle$ is easily expressed as

$$
\begin{aligned}
\left\langle\mathbf{u}(\tau)^{2}\right\rangle= & \frac{1}{Z} \int d \mathbf{u}_{f} \int d \mathbf{u}_{i} \int d \mathbf{u}_{1} K\left(\mathbf{u}_{1}, \mathbf{u}_{i} ; \tau,-N / 2\right) \\
& \times \mathbf{u}_{1}^{2} K\left(\mathbf{u}_{f}, \mathbf{u}_{1} ; N / 2, \tau\right),
\end{aligned}
$$

where $Z$ is identical to the numerator except for the absence of $\mathbf{u}_{1}^{2}$. In general, the propagator $K\left(\mathbf{u}_{f}, \mathbf{u}_{i} ; N / 2,-N / 2\right)$ can be evaluated by minimizing the action $S[\mathbf{u}]$, i.e., by finding the trajectory $\overline{\mathbf{u}}(\tau)$ such that $\delta S[\mathbf{u}] / \delta \mathbf{u}=0$. The required trajectory satisfies

$$
\frac{\partial^{2} \overline{\mathbf{u}}(\tau)}{\partial \tau^{2}}-\frac{2 \nu}{\epsilon} \overline{\mathbf{u}}(\tau)=0,
$$

whose solution, when substituted into Eq. (A2), produces the following expression for the minimized action:

$$
\begin{aligned}
S[\overline{\mathbf{u}}]= & \left(\frac{\nu \boldsymbol{\epsilon}}{2}\right)^{1 / 2} \frac{1}{\sinh \left((2 \nu / \epsilon)^{1 / 2} N\right)}\left[\left(\mathbf{u}_{i}^{2}+\mathbf{u}_{f}^{2}\right)\right. \\
& \left.\times \cosh \left(\left(\frac{2 \nu}{\epsilon}\right)^{1 / 2} N\right)-2 \mathbf{u}_{i} \cdot \mathbf{u}_{f}\right] .
\end{aligned}
$$

Since $S$ is quadratic in $\mathbf{u}$, the substitution $\mathbf{u}(\tau) \rightarrow \mathbf{u}(\tau)$ $+\overline{\mathbf{u}}(\tau)$ in Eq. (A1) leads to

$$
K\left(\mathbf{u}_{f}, \mathbf{u}_{i} ; N / 2,-N / 2\right)=\exp [-S[\overline{\mathbf{u}}]] K(0,0 ; N / 2,-N / 2) .
$$


Having determined the propagator, $\left\langle\mathbf{u}(\tau)^{2}\right\rangle$ is now trivially calculated from Eq. (A3) by carrying out the integrations over $\mathbf{u}_{i}, \mathbf{u}_{f}$, and $\mathbf{u}_{1}$. This yields the result

$$
\begin{aligned}
\left\langle\mathbf{u}(\tau)^{2}\right\rangle & \\
= & \left(\frac{9}{2 \nu \epsilon}\right)^{1 / 2} \\
& \times \frac{\cosh \left((2 \nu / \epsilon)^{1 / 2}(N / 2+\tau)\right) \cosh \left((2 \nu / \epsilon)^{1 / 2}(N / 2-\tau)\right)}{\sinh \left((2 \nu / \epsilon)^{1 / 2} N\right)} .
\end{aligned}
$$

A final integration over $\tau$ produces the first term of Eq. (46).

${ }^{1}$ T. L. Hill, An Introduction to Statistical Thermodynamics (Dover, New York, 1986.); B. Gaveau and L. S. Schulman, Phys. Rev. A 42, 3470 (1990).

${ }^{2}$ P. S. Doyle, B. Ladoux, and J.-L. Viovy, Phys. Rev. Lett. 84, 4769 (2000); X. Châtelier, T. J. Senden, J.-F. Joanny, and J.-M. Di Meglio, Europhys. Lett. 41, 303 (1998); H. Jensenius and G. Zocchi, Phys. Rev. Lett. 79, 5030 (1997); M. Reif, F. Osterhelt, B. Heymann, and H. E. Gaub, Science 275, 1295 (1997); P. Cluzel, A. Lebrun, C. Heller et al., ibid. 271, 792 (1996); S. B. Smith, L. Finzi, and C. Bustamante, ibid. 258, 1122 (1992).

${ }^{3}$ D. E. Smith, H. P. Babcock, and S. Chu, Science 283, 1724 (1999).

${ }^{4}$ L. Li and R. G. Larson, Macromolecules 33, 1411 (2000); P. S. Doyle, E. S. G. Shaqfeh, and A. P. Gast, J. Fluid Mech. 334, 251 (1997); J. J. López Cascales, F. G. Díaz, and J. García de la Torre, ibid. 36, 345 (1995).

${ }^{5}$ See, for example, P.-G. de Gennes, J. Chem. Phys. 60, 5030 (1974).

${ }^{6}$ A. V. Lyulin, D. B. Adolf, and G. R. Davies, J. Chem. Phys. 111, 758 (1999).
${ }^{7}$ A. Dua and B. J. Cherayil, J. Chem. Phys. 112, 8707 (2000).

${ }^{8}$ R. B. Bird, R. C. Armstrong, and O. Hassager, Dynamics of Polymeric Liquids (Wiley, New York, 1987); J. M. Wiest, L. E. Wedgewood, and R. B. Bird, J. Chem. Phys. 90, 587 (1989); A. Peterlin, J. Polym. Sci., Polym. Chem. Ed. 4, 287 (1966); Makromol. Chem. 44-46, 338 (1961).

${ }^{9}$ Y. Oono and K. F. Freed, J. Chem. Phys. 75, 1009 (1981); Y. Oono, Adv. Chem. Phys. 61, 301 (1985); A. Jagannathan, B. Schaub, and Y. Oono, Phys. Lett. A 113, 341 (1985); S. Puri, B. Schaub, and Y. Oono, ibid. 114, 399 (1986); S.-Q. Wang and K. F. Freed, J. Chem. Phys. 88, 3944 (1988); Y. Rabin, ibid. 88, 4014 (1988); S.-Q. Wang, Phys. Rev. A 40, 2137 (1989).

${ }^{10}$ K. Freed, Adv. Chem. Phys. 22, 1 (1972).

${ }^{11}$ T. Maeda and S. Fujime, Macromolecules 14, 809 (1981).

${ }^{12}$ R. A. Harris and J. E. Hearst, J. Chem. Phys. 44, 2595 (1966).

${ }^{13}$ R. G. Winkler, P. Reineker, and L. Harnau, J. Chem. Phys. 101, 8119 (1994); L. Harnau, R. G. Winkler, and P. Reineker, ibid. 102, 7750 (1995); W. Carl, J. Polym. Sci., Part B: Polym. Phys. 36, 1995 (1998).

${ }^{14}$ B. J. Cherayil and K. F. Freed, J. Chem. Phys. 104, 5983 (1996).

${ }^{15}$ See, for example, H. Hochstadt, Differential Equations, A Modern Approach (Dover, New York, 1975).

${ }^{16}$ L. Li and R. G. Larson, Macromolecules 33, 1411 (2000).

${ }^{17}$ B. H. Zimm, Macromolecules 31, 6089 (1998).

${ }^{18} \mathrm{X}$. Wang and A. P. Chatterjee (preprint), Macromolecules (submitted).

${ }^{19}$ L. Harnau, R. G. Winkler, and P. Reineker, J. Chem. Phys. 104, 6355 (1996).

${ }^{20}$ S.-Q. Wang, J. F. Douglas, and K. F. Freed, Macromolecules 18, 2464 (1985); J. Chem. Phys. 85, 3674 (1986).

${ }^{21}$ S. R. Quake, H. Babcock, and S. Chu, Nature (London) 388, 151 (1997).

${ }^{22}$ K. Soda, J. Phys. Soc. Jpn. 35, 866 (1973); S. R. Aragón and R. Pecora, Macromolecules 18, 1868 (1985).

${ }^{23}$ J. B. Lagowski and J. Noolandi, J. Chem. Phys. 95, 1266 (1991). 